\title{
Cranberry proanthocyanidins have anti-biofilm properties against Pseudomonas aeruginosa
}

\author{
Robert K Ulrey ${ }^{1}$, Stephanie M Barksdale ${ }^{2}$, Weidong Zhou ${ }^{3}$ and Monique L van Hoek ${ }^{2,4^{*}}$
}

\begin{abstract}
Background: Bacteria within a biofilm are phenotypically more resistant to antibiotics, desiccation, and the host immune system, making it an important virulence factor for many microbes. Cranberry juice has long been used to prevent infections of the urinary tract, which are often related to biofilm formation. Recent studies have found that the A-type proanthocyanidins from cranberries have anti-biofilm properties against Escherichia coli.

Methods: Using crystal violet biofilm staining, resazurin metabolism assays, and confocal imaging, we examined the ability of A-type proanthocyanidins (PACs) to disrupt the biofilm formation of Pseudomonas aeruginosa. We used mass spectrometry to analyze the proteomic effects of PAC treatment. We also performed synergy assays and in vitro and in vivo infections to determine whether PACs, alone and in combination with gentamicin, could contribute to the killing of $P$. aeruginosa and the survival of cell lines and G. mellonella.

Results: Cranberry PACs reduced P. aeruginosa swarming motility. Cranberry PACs significantly disrupted the biofilm formation of $P$. aeruginosa. Proteomics analysis revealed significantly different proteins expressed following PAC treatment. In addition, we found that PACs potentiated the antibiotic activity of gentamicin in an in vivo model of infection using G. mellonella.

Conclusions: Results suggest that A-type proanthocyanidins may be a useful therapeutic against the biofilm-mediated infections caused by P. aeruginosa and should be further tested.
\end{abstract}

Keywords: Cranberry, Proanthocyanidins, Pseudomonas aeruginosa, Biofilm

\section{Background}

Biofilms are colonies of bacteria encased within extracellular polymeric matrix [1]. Sessile biofilm bacteria are phenotypically different than planktonic bacteria, conferring increased resistance to desiccation, antibiotics, and the immune response. Antibiotics are able to kill the planktonic cells released by the biofilm after its maturation stages, but bacteria within the biofilm can persist, causing chronic infections [2]. In biofilm formation, bacteria attach reversibly to a surface, and then begin to produce extracellular polysaccharides. As the bacterial number grows, quorum sensing allows a phenotypic change in the bacteria. The biofilm matures and grows.

\footnotetext{
* Correspondence: mvanhoek@gmu.edu

${ }^{2}$ School of Systems Biology, George Mason University, Manassas, Virginia, USA

${ }^{4}$ National Center for Biodefense and Infectious Diseases, George Mason University, Manassas, Virginia, USA

Full list of author information is available at the end of the article
}

Eventually, proteins break down parts of the matrix so that bacteria within the biofilm can disperse [2].

Cranberry juice has long been used to prevent infections of the urinary tract, which are often related to biofilm formation [3-5]. Recent studies have found that the A-type proanthocyanidins from cranberries have antibiofilm properties against Escherichia coli [6,7]. The primary active compound in cranberries is the condensed tannin A-type proanthocyanidins (PACs), which comprises about $65 \%$ of cranberry non-dialyzable material (NDM) [8]. This oligomer is comprised of several types of alpha-linked flavan-3-ols that are substituted variously with hydroxyls along the aromatic and fused oxytane rings, as shown in Figure 1. It has been found that Atype PACs are iron chelators, indicating that PACs may disrupt normal bacterial function by limiting the supply of iron [9]. PACs have been shown to prevent adhesion and reduce biofilm production by a variety of pathogens [10-14]. Research has revealed the ability of PACs to 


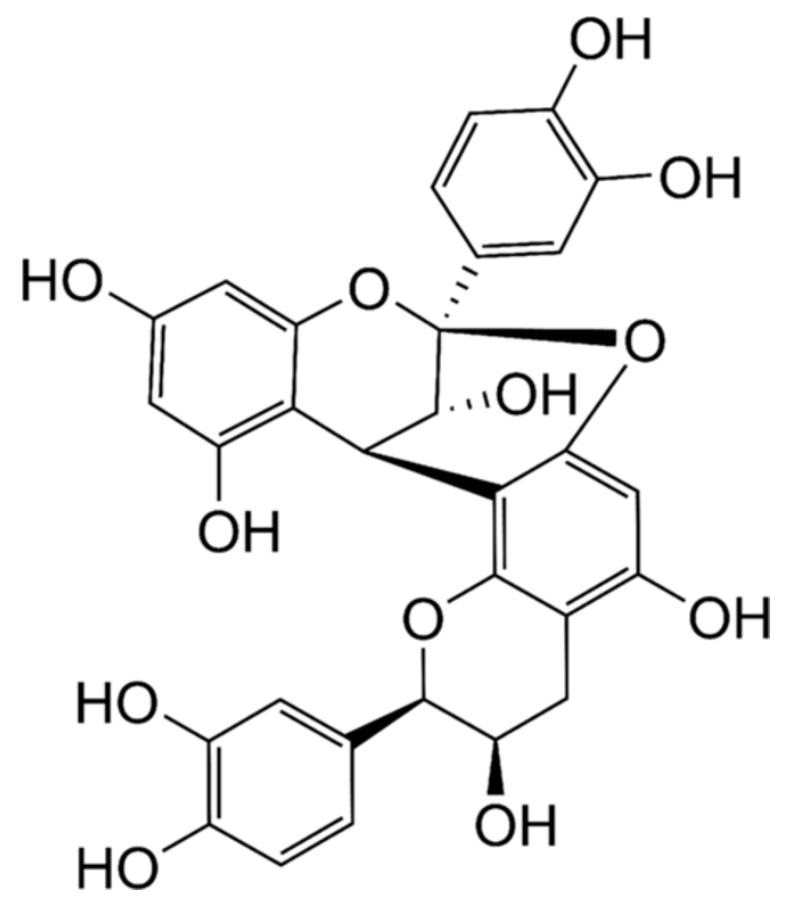

Figure 1 Structure of the A-type proanthocyanidin monomer. This structure represents the published chemical structure of PAC, the active ingredient in cranberry extract [8].

prevent the P-fimbriae adhesion of Escherichia coli in vitro and in vivo [15]. It has been shown that the alpha linkages in the compounds seem to be necessary to prevent adhesion [7]. Researchers have also found that PACs impair flagellum-mediated motility by Pseudomonas aeruginosa [16]. Thus, cranberry contains a variety of flavonols and proanthocyanidins that can be isolated and assessed for antimicrobial and anti-virulence activity [14,17-22].

$P$. aeruginosa is a Gram negative, opportunistic human pathogen associated with colonization and infection of vital organs such as the lungs, urinary tract, and kidneys [23]. This is a serious issue for cystic fibrosis patients, for whom $P$. aeruginosa colonization in the lung is the leading cause of illness and death [24]. P. aeruginosa is associated with many hospital-acquired infections due to colonization of medical equipment. Infection is usually associated with sepsis and general inflammation. This bacterium is also implicated in the mixed biofilm infections of burn victims, chronic wounds and diabetic pressure ulcers [25]. Biofilm formation is a major virulence factor in $P$. aeruginosa, and treatments that address this element of its pathogenicity are greatly needed.

In these experiments, we studied the ability of cranberry PACs to inhibit biofilm formation of $P$. aeruginosa, potentiate the activity of gentamicin, as well as the ability of PACs to lessen Pseudomonas pathogenicity in vitro and in vivo. The identification of natural products that inhibit the biofilm formation of $P$. aeruginosa and other important pathogens of humans is of great interest.

\section{Methods}

Bacteria and materials

P. aeruginosa ATCC 9027 was obtained from the American Type Culture Collection (Manassas, VA). GFP-expressing $P$. aeruginosa (Strain: PAO1-pTDK-GFP) was a generous gift from Douglas Weibel from the University of Wisconsin (Madison, WI). Both strains were grown overnight in Tryptic Soy Broth (TSB, Difco 211825) in a shaking incubator at $37^{\circ} \mathrm{C}$, bacterial pellets frozen in $20 \%$ glycerol at $-80^{\circ} \mathrm{C}$, and enumerated via serial dilution on Tryptic Soy Agar (Difco 236950). Cranberry PACs were generously provided by A. Howell (Rutgers University, NJ) and Ocean Spray.

\section{Swarming motility assay}

An assessment of the swarming motility of $P$. aeruginosa strain PAO1 was performed as previously described with minor modification [26]. TSB alone or with $100 \mu \mathrm{g} / \mathrm{mL}$ PACs was inoculated with overnight grown bacterial stocks. Overnight growth was adjusted with sterile 1X PBS to $\mathrm{OD}_{600}$ 0.6. Petri dishes were filled with about $15 \mathrm{~mL}$ of modified M9 medium with $0.5 \%$ granulated agar (Difco 214530) and allowed to dry under laminar flow for $60 \mathrm{~min} .5 \mu \mathrm{L}$ of bacterial inoculum was placed in the center of the plate. Plates were incubated for $16 \mathrm{~h}$ at $30^{\circ} \mathrm{C}$. Inhibition of swarming was measured qualitatively.

\section{Biofilm production inhibition assay}

Biofilm inhibition by cranberry PACs was measured by the crystal violet stain method with some alterations [27]. P. aeruginosa ATCC $9027\left(10^{5} \mathrm{CFU}\right)$ in TSB was incubated with varying concentrations of PACs, or without treatment, in triplicate in a 96-well plate (Corning $353072)\left(24 \mathrm{~h} ; 37^{\circ} \mathrm{C}\right)$. Initial turbidity was measured at $\mathrm{OD}_{600}$. Wells were washed with tap water, and the biofilm fixed at $60^{\circ} \mathrm{C}$ in a hybridization oven for 1 hour. The plate was stained with $0.1 \%$ crystal violet for 5 minutes, washed, and reconstituted with $33 \%$ acetic acid. The plate was read on a spectrophotometer at $\mathrm{OD}_{590}$. Student's $t$-test was performed between the growth/biofilm ratio for each experimental value and the negative control, as determined by absorbance at $\mathrm{OD}_{600}$ and $\mathrm{OD}_{590}$.

\section{Disruption of pre-formed biofilm}

The disruption of pre-formed biofilm by cranberry PACs was assayed using $P$. aeruginosa ATCC 9027 as previously described [25,28]. After biofilm formation (24 h, $37^{\circ} \mathrm{C}$ ) in a 96-well plate (Corning, 353072), various concentrations of PACs were added to the wells in triplicate. Wells without treatment served as the negative control. After an additional incubation $\left(24 \mathrm{~h}, 37^{\circ} \mathrm{C}\right)$, bacterial 
growth was measured at $\mathrm{OD}_{600}$, and the crystal violet stain was performed as above in the biofilm production inhibition assay.

\section{Bacterial attachment assay}

Attachment assays were performed in a 96-well microtiter plate (Corning, 353072) as previously described [25,28]. Overnight cultures of $P$. aeruginosa ATCC 9027 were grown in TSB to an $\mathrm{OD}_{600}$ of $\sim 1.0$. Bacterial culture was added to wells containing varying concentrations of PACs, or no PACs in triplicate. The plates were incubated $(1 \mathrm{~h}$, $37^{\circ} \mathrm{C}$ ) to allow attachment and the absorbance was measured at $\mathrm{OD}_{600}$. The crystal violet stain was performed as for the biofilm production assay [29].

\section{MIC and checkerboard assays}

The minimal inhibitory concentration of gentamicin for P. aeruginosa ATCC 9027 was determined as previously in Mueller Hinton Broth [30]. A checkerboard assay was performed as previously described [31,32].

\section{Confocal imaging of flow cell slides}

The flow chamber was set up and inoculated as previously described, with some modifications [33]. Frozen $\mathrm{GFP}^{+}$P. aeruginosa $\left(2 \times 10^{9}\right.$ CFUs $)$ was incubated for $1 \mathrm{~h}$ at $37^{\circ} \mathrm{C}$ in TSB and then was injected in a flow cell unit (FC 284 Dual Channel Transmission Flow Cell, BioSurface Technologies Corp.) with TSB and $100 \mu \mathrm{g} / \mathrm{mL}$ ampicillin. PACs $(10 \mu \mathrm{g}$ in $1 \mathrm{~mL})$ were injected simultaneously with the bacteria. Untreated GFP+ $P$. aeruginosa was used as a control. Slides were imaged with a Nikon TE-2000 confocal using Z-stack. Graphics generated using Nikon EZ-C1 software. The experiment was performed in duplicate.

\section{Resazurin metabolism measurement assay}

P. aeruginosa ATCC 9027 was grown in a 96-well plate (Corning 353072) and treated with PACs in various concentrations, in the same manner as the biofilm production inhibition assay. After $24 \mathrm{~h}$ growth, media was aspirated, and wells washed with $1 \mathrm{X}$ PBS. Resazurin $(0.0064 \%)$ in TSB-C was added to each well. The $\mathrm{OD}_{590}$ was read kinetically in a spectrophotometer $(30 \mathrm{~min}$; $37^{\circ} \mathrm{C}$ ). The quantitative difference in metabolic reduction of resazurin was compared at the 15 min time point between experimental and control wells with a Student's $t$-test.

\section{Calgary device}

The Calgary device (Innovatech) HTP MBEC assay was performed according to manufacturer's instructions with small changes. Bacteria $\left(10^{5} \mathrm{CFU} /\right.$ well $)$ were exposed to varying concentrations of PACs in TSB in the Calgary device plate and incubated for $24 \mathrm{~h}$ at $37^{\circ} \mathrm{C}$ ). Pegs on the plate lid were washed twice in fresh 96-well plates with sterile PBS and sealed thoroughly on a third plate containing PBS. This plate was sonicated for $30 \mathrm{~min}$ with the sealed plate sitting on the surface of a water-bath sonicator, and the resulting disrupted biofilm was plated in triplicate via serial dilution on TSA plates. Experiment was repeated 6 times. Experimental and control CFU counts were compared via Student's $t$-test.

\section{Proteomic analysis}

Treated and untreated $P$. aeruginosa was grown overnight in TSB at $37^{\circ} \mathrm{C}$ in a shaking incubator, with or without $100 \mu \mathrm{g} / \mathrm{mL}$ PACs. $1 \mathrm{~mL}$ was centrifuged to pellet $(13,200 \mathrm{rpm}, 10 \mathrm{~min})$ and resuspended in $1 \mathrm{~mL}$ distilled water. Bacteria were lysed by repeated freeze-thaw cycles. Protein content was measured by the BCA protein assay (Pierce) and samples adjusted to equivalent protein concentrations with sterile water. $100 \mu \mathrm{g}$ of protein were reduced by $10 \mathrm{mM}$ dithiothreitol (DTT) for 30 minutes at $37^{\circ} \mathrm{C}$, and then alkylated by $50 \mathrm{mM}$ iodoacetamide for $20 \mathrm{~min}$ at room temperature. The proteins were digested by trypsin at $37^{\circ} \mathrm{C}$ for $6 \mathrm{~h}$ in a buffer containing ammonium bicarbonate $(50 \mathrm{mM}, \mathrm{pH} 9)$ and $2 \mathrm{M}$ urea. The digestion mixture was then acidified by adding glacial acetic acid to a final concentration of $2 \%$ and desalted by ZipTip (Millipore). The peptides were analyzed by high sensitive LC-MS/MS using an LTQOrbitrap mass spectrometer (Thermo Fisher) as previously described [34]. Tandem mass spectra collected by Xcalibur (version 2.0.2) were searched against the NCBI $P$. aeruginosa PAO1 protein database using SEQUEST (Bioworks software from ThermoFisher, version 3.3.1) with full tryptic cleavage constraints, static cysteine alkylation by iodoacetamide, and variable methionine oxidation. Mass tolerance for precursor ions was $5 \mathrm{ppm}$ and mass tolerance for fragment ions was $0.25 \mathrm{Da}$. The SEQUEST search results of proteomics data were filtered by the criteria "Xcorr versus charge 1.9, 2.2, 3.0 for $1+$, $2+, 3+$ ions; $\Delta \mathrm{Cn}>0.1$; ranked top $\# 1$; probability of randomized identification of peptide $<0.01$ ". Confident peptide identifications were determined using these stringent filter criteria for database match scoring followed by manual evaluation of the results. The "false discovery rate (FDR)" was estimated by searching a combined forward-reversed database as described by Elias [35]. The SEQUEST search results were exported to spreadsheets and compared. Pathway classifications were done manually using the Pseudomonas genomic database (pseudomonas.org) and the KEGG Pathway module.

\section{Treatment of infected mammalian cells with PACs}

J774A.1 mouse macrophage cells (ATCC TIB-67) and HEK293T/17 human kidney epithelial cells (ATCC CRL11268) were acquired from the American Type Culture 
Collection (Manassas, VA), and were grown from a frozen stock with limited passaging. Cells were grown in Dulbecco's Modified Eagle Medium (DMEM) with 10\% Fetal Bovine Serum (FBS) and 1\% penicillin/streptomycin. Cells were pelleted, washed once with PBS, pelleted again, and then resuspended in DMEM with 10\% FBS. A tissue culture-treated 96-well plate (Corning 353072) was seeded with $5 \times 10^{4}$ cells per well. P. aeruginosa ATCC 9027 at a multiplicity of infection (MOI) of 500 was added for $15 \mathrm{~h}$. PACs $(10 \mu \mathrm{g} / \mathrm{mL})$ were added to experimental wells simultaneously with the bacteria. Non-infected cells were used to establish a spontaneous $\mathrm{LDH}$ release control. $\mathrm{LDH}$ release was measured kinetically for 15-17.5 hours using a spectrophotometer at $\mathrm{OD}_{490}$. The readings of treated wells were compared at the $15 \mathrm{~h}$ time-point with control wells using a Student's t-test. Controls and experimental wells were set up in triplicate and the assay was performed 3 times.

\section{In vivo treatment of infected Galleria mellonella (wax moth larvae)}

G. mellonella larvae (waxworms) were purchased from Vanderhorst Wholesale (St. Mary's, OH) and used as a model of bacterial infection as previously described [25]. All injections were done into the first proleg unless the first proleg was deformed. The second injection was into the second proleg if 2 injections were performed. The waxworms weighed between 0.23-0.38 g. Tuberculin needles $(0.5 \mathrm{~mL})$ were used for injections. The waxworms were stored in plastic petri dishes at $37^{\circ} \mathrm{C}$ after injection and assessed for vital signs daily $[25,30,36]$.

\section{Statistical analysis}

The means and the standard deviation were calculated for the indicated experiments. The statistical analysis performed was Student's $t$-test with a level of significance of $\mathrm{p}<0.05$.

\section{Results and discussion}

\section{PACs reduced $P$. aeruginosa motility}

A previous study found that cranberry PACs inhibited the swarming motility of P. aeruginosa [16]. Our experiments confirmed that the swarming motility of $P$. aeruginosa was limited with the addition of $100 \mu \mathrm{g} / \mathrm{mL}$ PACs (Figure 2). Untreated bacteria swarmed to the edge of the agar plate (Figure 2A), while bacteria growing on PACcontaining agar moved about half the distance (Figure 2B). In addition, swarming patterns were not as branched or complex.

\section{Effect of cranberry PACs on $P$. aeruginosa biofilm}

Previous studies of both Gram-positive and Gram-negative bacteria have shown a significant decrease in biofilm production when exposed to cranberry juice, cranberry

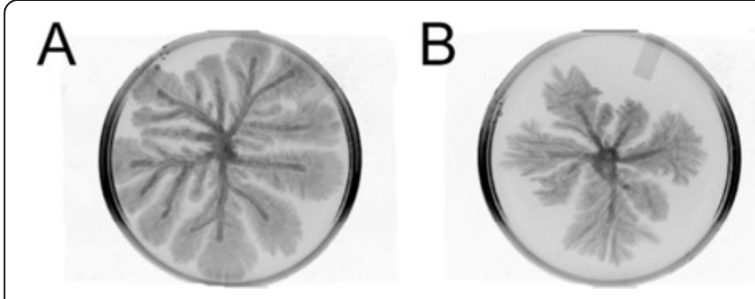

Figure 2 Photographs of $P$. aeruginosa swarming motility on modified M9 agar, with A. modified M9 agar and B. modified M9 agar with $100 \mu \mathrm{g} / \mathrm{mL}$ cranberry PACs added.

extracts, and cranberry PACs [10-14]. In our experiments, cranberry PACs significantly inhibited biofilm formation of $P$. aeruginosa in vitro at concentrations as low as $1 \mu \mathrm{g} / \mathrm{mL}(\mathrm{p}<0.05)$ (Figure $3 \mathrm{~A})$. At $1 \mu \mathrm{g} / \mathrm{mL}$, PACs inhibited biofilm formation $40.9 \%$, while $10 \mu \mathrm{g} / \mathrm{mL}$ PACs inhibited biofilm formation $55.7 \%(\mathrm{p}<0.01)$ compared to the untreated $P$. aeruginosa.

When bacteria were allowed to attach and form biofilm for $24 \mathrm{~h}$ before treatment, exposure to $10 \mu \mathrm{g} / \mathrm{mL}$ PACs for an additional $24 \mathrm{~h}$ resulted in a $54.1 \%(\mathrm{p}<0.05)$ reduction of preformed biofilm (compared to untreated control) and at $100 \mu \mathrm{g} / \mathrm{mL}$, a $39.6 \%$ at $(\mathrm{p}<0.01)$ inhibition compared to the untreated control (Figure 3B). PAC treatment showed no significant effect on the attachment of $P$. aeruginosa (Figure $3 \mathrm{C}$ ). Using the Calgary device (consisting of inverted plastic pegs that hang down into the media of a 96 well plate, to which bacteria attach and form biofilm), exposure to PACs showed a $49.5 \%$ decrease in bacteria attached to the inverted pegs as compared to control at $100 \mu \mathrm{g} / \mathrm{mL}(\mathrm{p}<0.1)$, but no statistical difference at any other concentration (Figure 3D), confirming the preformed biofilm results above.

In confocal imaging experiments in support of Figure 3A, imaged after the incubation of bacteria with PACs in a flow cell apparatus, it was found that biofilm height appeared to decrease after PAC treatment. Figures 4A - D illustrate the change in the height of $P$. aeruginosa biofilm, which decreased from $\sim 26 \mu \mathrm{m}$ to $\sim 20 \mu \mathrm{m}$ when treated with $10 \mu \mathrm{g}$ PACs during attachment phase. In addition, the biofilm density also appeared to decrease.

\section{Proteomics of PAC-treated $P$. aeruginosa}

To understand changes in global protein expression of the bacterium in response to PACs treatment, $P$. aeruginosa was grown overnight with or without PACs $(100 \mu \mathrm{g} / \mathrm{mL})$, and then subjected to proteomic analysis by mass-spectrometry (LC-MS-MS) (3 replicates). A total of 1075 proteins were identified in the untreated sample, and 1144 proteins were identified in treated samples with 1\% FDR (Figure 5A). The expression levels of the identified proteins in the two samples were 

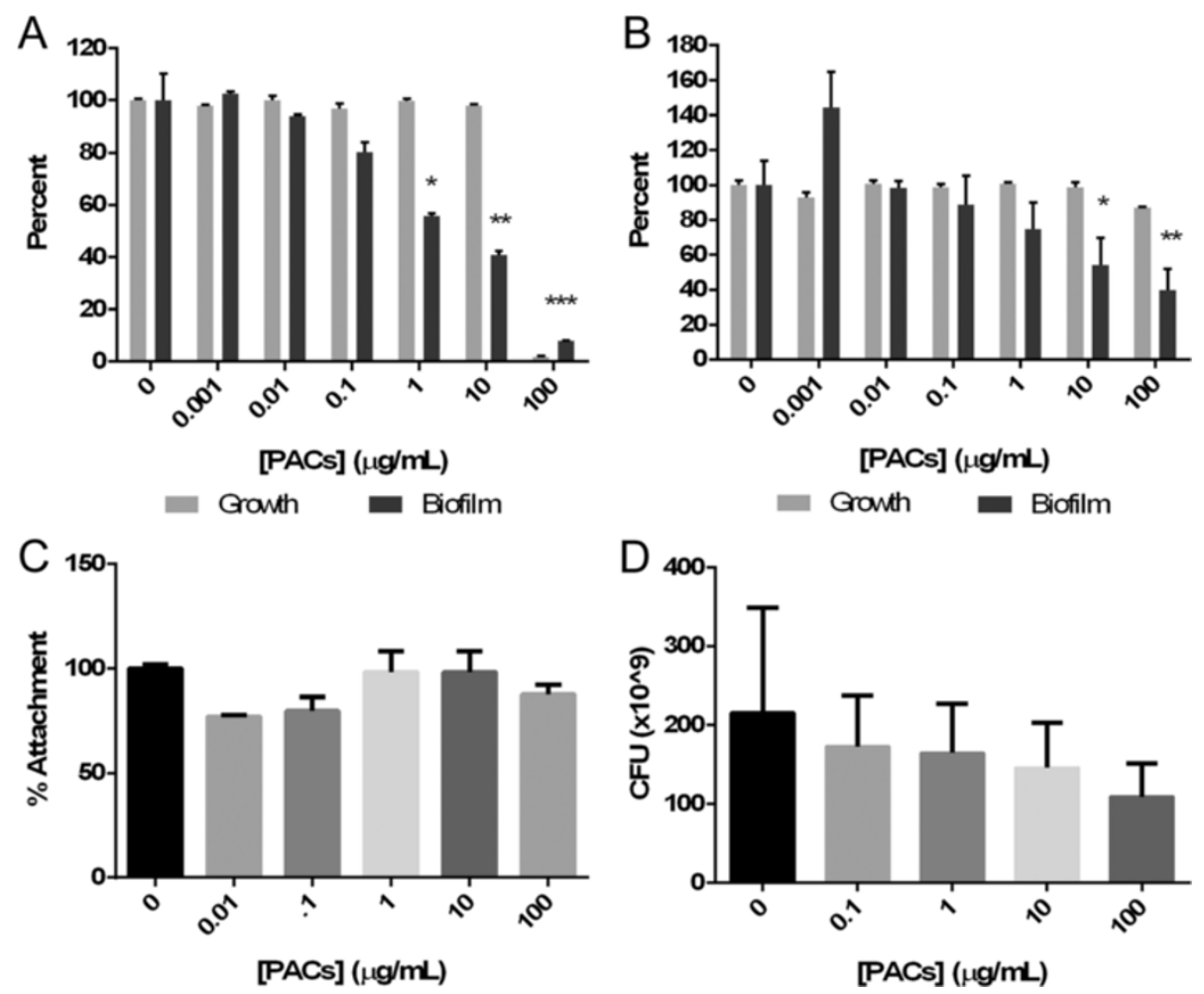

Figure 3 Effects of cranberry PACs on P. aeruginosa. A. biofilm formation (PACs dissolved in DMSO), B. pre-formed biofilm (PACs dissolved in deionized water), C. surface attachment, D. Bacterial number from treated and untreated biofilm using the Calgary device. ${ }^{*}=p<0.05 ;{ }^{* *}=p<0.005$; $*^{* *}=\mathrm{p}<0.001$.

compared by spectra count using the label-free quantitation method [34], and the result revealed many differentially expressed proteins (Additional file 1: Table S1).

Of 159 proteins that had the largest differences in abundance, the 30 most differentially regulated proteins were chosen from each group (up- and down-regulated) for examination in greater detail, as shown in Figure 5B. The top 30 proteins are listed in Table 1 (up-regulated) and Table 2 (down regulated). Proteins that were most significantly up-regulated (Table 1 ) include 12 proteins related to iron siderophores or cation transporters, for example, PchD, PvdN, and PhuS. 5 proteins putatively involved in amino acid synthesis were also up-regulated, including PA0335, PA2044, and HutG. In addition, several proteins that are up-regulated in response to stress were also found, such as OsmC and SodM. A hypothetical protein (PA3450) thought to be involved with flavonoid metabolism was also up-regulated.

A wide variety of proteins were down-regulated due to PACs treatment (Table 2). This included 2 proteins related to ATP synthesis, a likely cytochrome C (PA2482) and hypothetical protein PA2481, and several proteins involved in DNA and RNA synthesis, such as TopA, RplC, and Mfd. In addition, several citric acid cycle proteins, such as subunits of the acetyl-CoA carboxylase and fumarase, were found to be significantly reduced.

Though it was found that the presence of PACs inhibited swarming motility, no proteins related to flagella or type IV pili were found be down-regulated. The quorumsensing proteins in the las and $r h l$ regulons were not found to be differentially regulated when treated, except for PvdQ, a quorum quencher [37], which had significantly more peptide hits with PACs treatment. It has been suggested that inhibition of quorum-sensing may be a mechanism to block $P$. aeruginosa biofilm production $[37,38]$.

\section{Further effect of cranberry PACs on biofilm-resident $P$. aeruginosa}

Metabolism in bacteria, as a measure of the number of bacteria present, can be measured with the compound resazurin, which becomes highly fluorescent when reduced to resorufin by chemicals or living cells, primarily by NADH oxidoreductases in bacteria. Biofilm-resident bacteria formed in the presence of higher concentrations of cranberry PACs had lower metabolic rates than biofilm-resident bacteria formed at lower concentrations of PACs (Figure 6). P. aeruginosa treated with $100 \mu \mathrm{g} / \mathrm{mL}$ PACs reduced $32.9 \%$ of the resazurin compared to the 

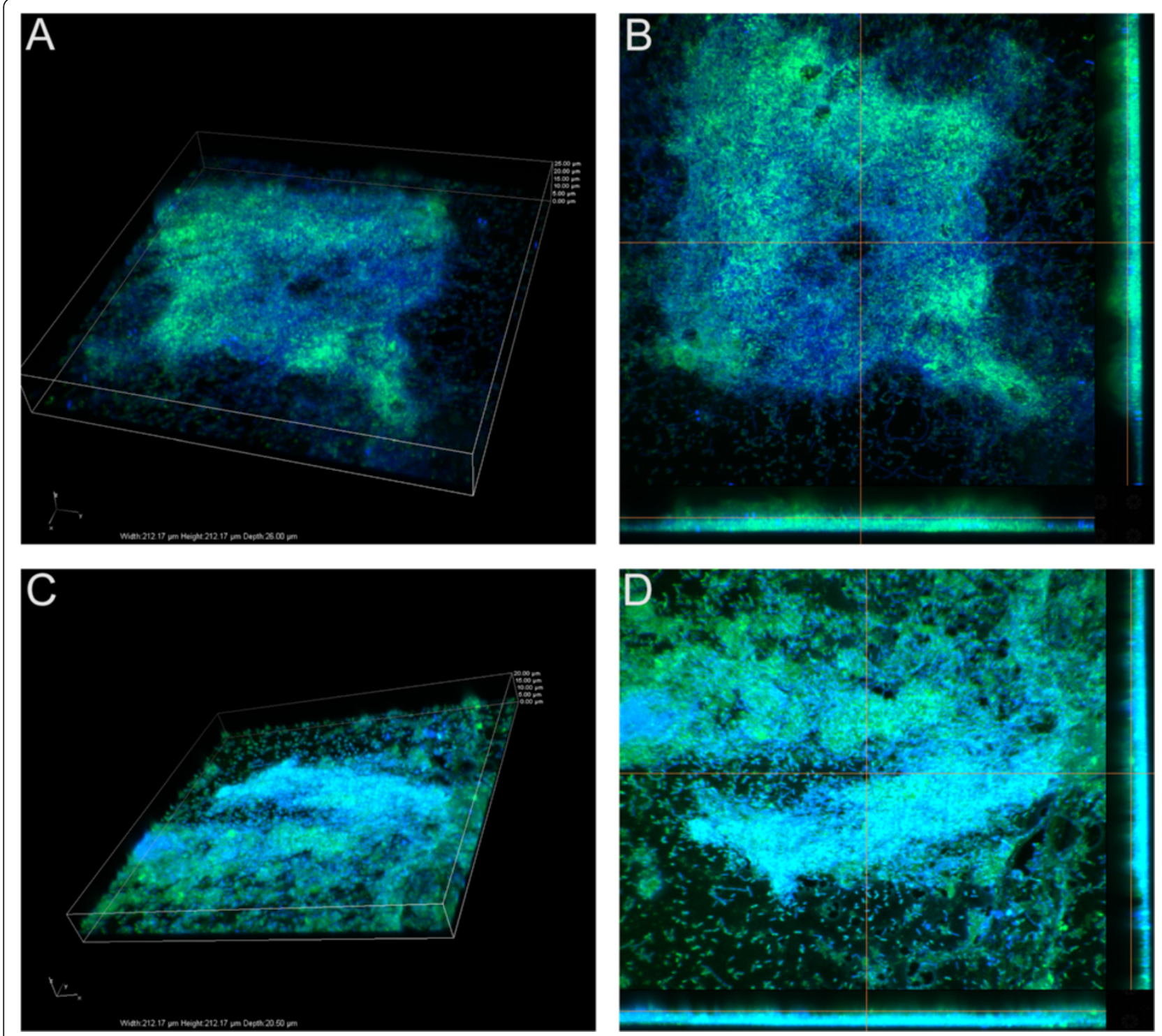

Figure 4 Confocal imaging (3D Z stack and sections) of $P$. aeruginosa biofilm. A. Untreated: 3-dimensional Z stack after $24 \mathrm{~h}$ incubation without PACs, B. Sections view after $24 \mathrm{~h}$ incubation without PACs, C. Treated: 3-dimensional Z stack after $24 \mathrm{~h}$ incubation with PACs, D. Sections view after 24 incubation with PACs.

untreated bacteria $(\mathrm{p}<0.01)$. At PAC concentrations of $10 \mu \mathrm{g} / \mathrm{mL}$, PAC-treated $P$. aeruginosa biofilm reduced $36.9 \%$ of the untreated control ( $p<0.01)$. Thus, these results most likely reflect a decrease in the total number of bacteria remaining in the PACs-reduced biofilm. Several citric acid cycle and ATP synthesis proteins were down-regulated, so the effect of PACs-treatment on $P$. aeruginosa biofilm may also be partly due to an effect on bacterial metabolism.

\section{In vitro potentiation of gentamicin by PACs}

PAC is known to be an iron chelator [39], and some iron chelators have been shown to potentiate the activity of antibiotics, particularly aminoglycosides such as gentamicin and tobramycin [40]. To determine whether PACs may be able to contribute to potentiation of the antibiotic activity of gentamicin, a checkerboard assay was performed using varying concentrations of PAC against various concentrations of gentamicin. The PACs did not kill P. aeruginosa at any concentration tested, and thus are not directly antimicrobial. Gentamicin had an MIC of $1.5 \mu \mathrm{g} / \mathrm{mL}$ against this bacterial strain, which is in agreement with published values [41]. However, the MIC of gentamicin in combination with PACs was $1.3 \mu \mathrm{g} / \mathrm{mL}$, as shown in Table 3. Based on these results, we concluded that PACs slightly potentiate the effect of the gentamicin in vitro. Cranberry PACs may be considered as an adjuvant to gentamicin. 


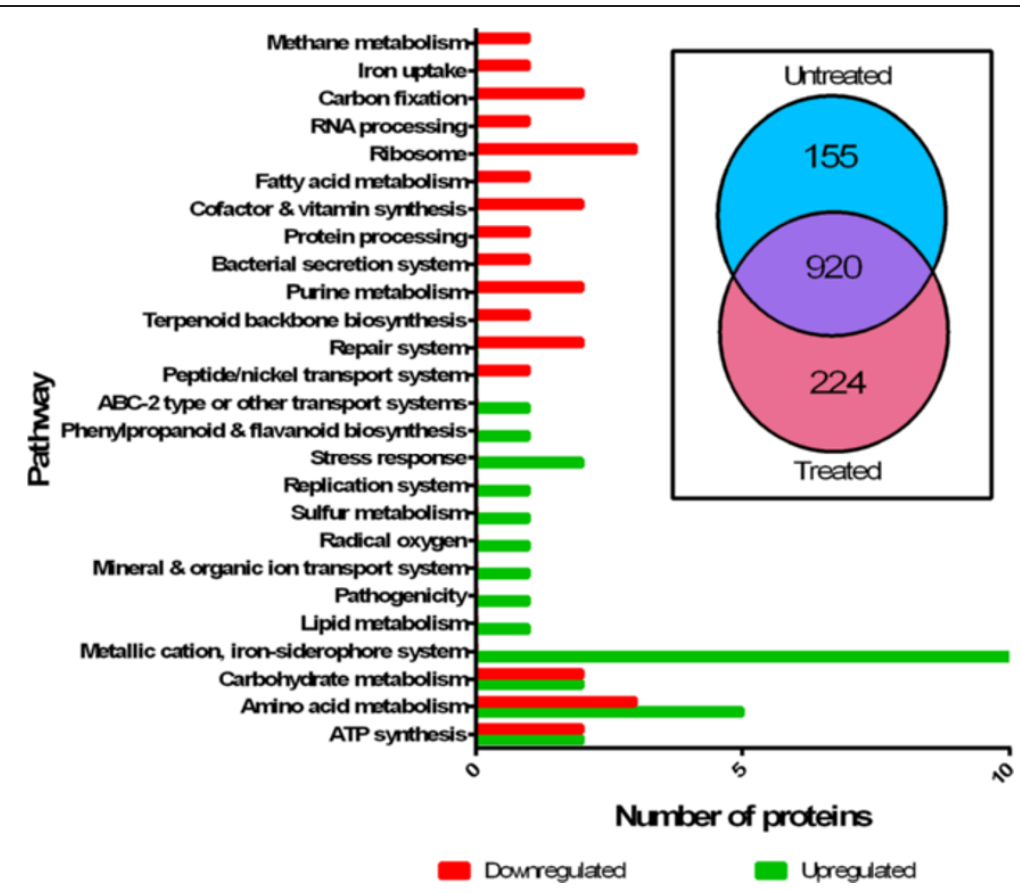

Figure 5 A proteomics analysis of PAC treated P. aeruginosa. The general function of the top 30 up-regulated and top 30 down-regulated proteins is illustrated, showing the general separation of function, and some overlapping functions between up- and down-regulated proteins. Inset: The number of proteins identified in the untreated and treated samples. Total of 1075 proteins were identified in untreated sample, and 1144 proteins were identified in treated samples. Total of 1299 proteins were identified in the two samples, and total of 920 common proteins were identified in both samples.

Treatment of $P$. aeruginosa-infected J774A.1 and HEK293T/17 cells with PACs

In order to determine if PACs treatment could rescue eukaryotic cells during a $P$. aeruginosa infection, murine macrophage cells (J774A.1) and human embryonic kidney cells (HEK293T/17) were first treated with varying concentrations of PACs, and then infected with P. aeruginosa $(\mathrm{MOI}=500)$ for 24 hours. The subsequent LDH release assay measured the release of lactate dehydrogenase, which is indicative of lysis. At $15 \mathrm{~h}$, it was found that $10 \mu \mathrm{g} / \mathrm{mL}$ PACs significantly rescued murine macrophages from $P$. aeruginosa-mediated lysis, as shown by reduced release of $\mathrm{LDH}$ compared to the untreated control ( $\mathrm{p}<0.05$, Figure 7A). This concentration of PACs also significantly rescued human embryonic kidney cells from lysis by $P$. aeruginosa ( $\mathrm{p}<0.05$, Figure $7 \mathrm{~B}$ ), with full kinetic data from the experiment shown.

Treatment of $P$. aeruginosa-infected G. mellonella with cranberry PACs in combination with gentamicin

Some potentiating effect was shown (Table 3 ) when gentamicin was combined with PACs. To see if this effect would occur in vivo, G. mellonella was infected with $P$. aeruginosa and treated with sub-MIC concentrations of gentamicin and low levels of PACs. G. mellonella waxworms are an in vivo infection model for multiple pathogens, including $P$. aeruginosa $[25,30,36]$. A KaplanMeier survival analysis conducted on the results obtained over a $72 \mathrm{~h}$ period (Figure 8 ) indicated that a combination therapy of PACs and gentamicin was significantly $(\mathrm{p}<0.05)$ more effective in reducing larvae death as compared to gentamicin treatment or PAC treatment alone. The average ratio of death over the $72 \mathrm{~h}$ time course between gentamicin alone, PAC alone, and gentamicin-PAC combination treatment was 3.4:1, suggesting the significant survival benefit of having the combination treatment.

\section{Conclusions}

PACs are phenolic oligomers found in relatively high abundance within cranberries. Recent studies have found that these polyphenol structures are effective in reducing biofilm formation [42]. Cranberry juice and extracts have historically been used to prevent urinary tract infections in women [3-5]. The extracted NDM of cranberry has been shown to prevent the formation of biofilm by S. epidermis on soft contact lenses, which could reduce ocular infections [43]. It has been shown previously that cranberry NDM contains primarily A-type PACs [43]. Cranberry NDM has been shown to interfere with quorum sensing in Vibrio harveyi [44]. Cranberry NDM has also been shown to reduce the colonization of Porphyromonas 
Table 1 The top 30 up-regulated proteins in PAC-treated $P$. aeruginosa bacteria

\begin{tabular}{|c|c|c|c|c|}
\hline \multicolumn{5}{|c|}{ Upregulated } \\
\hline \multirow[t]{2}{*}{ Proteins } & \multirow[t]{2}{*}{ Functional annotation } & \multirow{2}{*}{$\begin{array}{l}\text { Accession } \\
\text { number }\end{array}$} & \multicolumn{2}{|c|}{ Average spectra coun } \\
\hline & & & Untreated & Treated \\
\hline fumarate hydratase FumC1 & Central carbohydrate metabolism & 15599666 & 0.1 & 21.3 \\
\hline ABC transporter ATP-binding protein PA4595 & ABC-2 type or other transport systems & 15599791 & 0.1 & 19 \\
\hline antioxidant protein PA3450 & Phenylpropanoid and flavanoid biosynthesis & 15598646 & 0.1 & 18 \\
\hline adhesion protein PA2407 & $\begin{array}{l}\text { Metallic cation, iron-siderophore and vitamin } \\
\text { B12 transport system }\end{array}$ & 15597603 & 0.1 & 17 \\
\hline 3-oxo-C12-homoserine lactone acylase PvdQ & $\begin{array}{l}\text { Metallic cation, iron-siderophore and vitamin } \\
\text { B12 transport system }\end{array}$ & 15597581 & 0.1 & 16 \\
\hline pyochelin biosynthesis protein PchD & $\begin{array}{l}\text { Metallic cation, iron-siderophore and vitamin } \\
\text { B12 transport system }\end{array}$ & 15599424 & 0.1 & 15.3 \\
\hline hypothetical protein PA2410 & $\begin{array}{l}\text { Metallic cation, iron-siderophore and vitamin } \\
\text { B12 transport system }\end{array}$ & 15597606 & 0.1 & 13.3 \\
\hline hypothetical protein PA0335 & Serine and threonine metabolism & 15595532 & 0.1 & 12.3 \\
\hline protein PvdN & $\begin{array}{l}\text { Metallic cation, iron-siderophore and vitamin } \\
\text { B12 transport system }\end{array}$ & 15597590 & 0.1 & 11.3 \\
\hline hypothetical protein PA4328 & Stress protein & 15599524 & 0.1 & 11 \\
\hline hypothetical protein PA3931 & $\begin{array}{l}\text { Metallic cation, iron-siderophore and vitamin } \\
\text { B12 transport system }\end{array}$ & 15599126 & 0.1 & 10 \\
\hline hypothetical protein PA4657 & ATP synthesis & 15599852 & 0.1 & 9.7 \\
\hline osmotically inducible protein OsmC & Stress protein & 15595257 & 0.1 & 9 \\
\hline PmbA & Replication system & 15599668 & 0.1 & 8.3 \\
\hline sulfite reductase Cysl & Sulfur metabolism & 15597035 & 0.1 & 7.3 \\
\hline superoxide dismutase SodM & Stress protein & 15599664 & 0.1 & 6.7 \\
\hline hypothetical protein PA3250 & $\begin{array}{l}\text { Metallic cation, iron-siderophore and vitamin } \\
\text { B12 transport system }\end{array}$ & 15598446 & 0.1 & 6.7 \\
\hline hypothetical protein PA2699 & Histidine metabolism & 15597895 & 0.1 & 6.3 \\
\hline hypothetical protein PA2044 & Cysteine and methionine metabolism & 15597240 & 0.1 & 6.3 \\
\hline periplasmic polyamine binding protein PA0295 & Mineral and organic ion transport system & 15595492 & 0.1 & 6.3 \\
\hline heme/hemoglobin uptake outer membrane receptor PhuR & $\begin{array}{l}\text { Metallic cation, iron-siderophore and vitamin } \\
\text { B12 transport system }\end{array}$ & 15599904 & 0.1 & 6 \\
\hline Phus & $\begin{array}{l}\text { Metallic cation, iron-siderophore and vitamin } \\
\text { B12 transport system }\end{array}$ & 15599903 & 0.1 & 5.7 \\
\hline pyocin S5 & Pathogenicity & 15596182 & 0.1 & 5.7 \\
\hline carbohydrate kinase PA3579 & Lipid metabolism & 15598775 & 0.1 & 5.3 \\
\hline hypothetical protein PA5229 & Unknown function & 15600422 & 0.1 & 5 \\
\hline heme uptake outer membrane receptor HasR & $\begin{array}{l}\text { Metallic cation, iron-siderophore and vitamin } \\
\text { B12 transport system }\end{array}$ & 15598604 & 0.1 & 5 \\
\hline SpoVR family protein PA0586 & Stress protein & 15595783 & 0.1 & 5 \\
\hline hypothetical protein PA5359 & Carbohydrate metabolism & 15600552 & 0.1 & 4.7 \\
\hline $\mathrm{N}$-formylglutamate amidohydrolase HutG & Histidine metabolism & 15600284 & 0.1 & 4.3 \\
\hline amidotransferase PauD2 & Histidine metabolism & 15596939 & 0.1 & 4.3 \\
\hline
\end{tabular}

gingivalis and mixed biofilms of $P$. gingivalis and Fusobacterium nucleatum in periodontal sites $[45,46]$.

In this study, we determined that cranberry PACs have anti-biofilm activities against the gram-negative bacterium
P. aeruginosa. O'May and Tufenkji [47] showed that the sessile biofilm lifestyle of $P$. aeruginosa is bolstered by the presence of cranberry PACs, which limit motility, particularly swarming motility. Our experiments confirmed that 
Table 2 The top 30 down-regulated proteins in PAC treated P. aeruginosa bacteria

\begin{tabular}{|c|c|c|c|c|}
\hline \multicolumn{5}{|c|}{ Downregulated } \\
\hline \multirow[t]{2}{*}{ Proteins } & \multirow[t]{2}{*}{ Functional annotation } & \multirow[t]{2}{*}{$\begin{array}{c}\text { Accession } \\
\text { number }\end{array}$} & \multicolumn{2}{|c|}{$\begin{array}{l}\text { Average spectra } \\
\text { count }\end{array}$} \\
\hline & & & Untreated & Treated \\
\hline ABC transporter PA4502 & Peptide/nickel export system & 15599698 & 13.7 & 0 \\
\hline hypothetical protein PA2481 & ATP synthesis & 15597677 & 8 & 0 \\
\hline DNA topoisomerase I TopA & Repair system & 15598207 & 7.7 & 0 \\
\hline homogentisate 1,2-dioxygenase HmgA & Aromatic amino acid metabolism & 15597205 & 16.3 & 0.1 \\
\hline $\begin{array}{l}\text { 4-hydroxy-3-methylbut-2-en-1-yl diphosphate synthase } \\
\text { GcpE }\end{array}$ & Terpenoid backbone biosynthesis & 15598998 & 11.3 & 0.1 \\
\hline inosine 5'-monophosphate dehydrogenase GuaB & Purine metabolism & 15598965 & 6.7 & 0.1 \\
\hline preprotein translocase subunit SecA & Bacterial secretion system & 15599599 & 6.7 & 0.1 \\
\hline hypothetical protein PA5545 & Protein processing & 15600738 & 6.7 & 0.1 \\
\hline $\begin{array}{l}\text { adenosylmethionine-8-amino-7-oxononanoate } \\
\text { aminotransferase BioA }\end{array}$ & Cofactor and vitamin synthesis & 15595617 & 6.3 & 0.1 \\
\hline hypothetical protein PA2765 & Unknown function & 15597961 & 6.3 & 0.1 \\
\hline 3-ketoacyl-CoA thiolase FoaB & Fatty acid metabolism & 15598209 & 6 & 0.1 \\
\hline acetyl-CoA carboxylase subunit beta AccD & Carbon fixation & 15598308 & 5.7 & 0.1 \\
\hline 30 S ribosomal protein S3 RpsC & Ribosome & 15599453 & 5.7 & 0.1 \\
\hline ribonuclease E Rne & RNA processing & 15598172 & 5.3 & 0.1 \\
\hline bacterioferritin PA4880 & $\begin{array}{l}\text { Metallic cation, iron-siderophore and vitamin B12 } \\
\text { xport system }\end{array}$ & 15600073 & 5 & 0.1 \\
\hline $50 S$ ribosomal protein L13 RplM & Ribosome & 15599629 & 4.7 & 0.1 \\
\hline formate dehydrogenase subunit epsilon FdhE & Other carbohydrate metabolism & 15600003 & 4.7 & 0.1 \\
\hline hypothetical protein PA3967 & Unknown function & 15599162 & 4.3 & 0.1 \\
\hline $\begin{array}{l}\text { lysine-specific pyridoxal 5'-phosphate-dependent } \\
\text { carboxylase LdcA }\end{array}$ & Arginine and proline metabolism & 15597015 & 4 & 0.1 \\
\hline acetyl-CoA carboxylase subunit A PA5436 & Citrate cycle & 15600629 & 4 & 0.1 \\
\hline cytochrome C PA2482 & ATP synthesis & 15597678 & 3.3 & 0.1 \\
\hline threonine dehydratase IIvA1 & Branched-chain amino acid metabolism & 15595528 & 3 & 0.1 \\
\hline transcription-repair coupling factor Mfd & Repair system & 15598198 & 3 & 0.1 \\
\hline glycerate dehydrogenase HprA & Photorespiration & 15599822 & 3 & 0.1 \\
\hline hypothetical protein PA5201 & Pathogenicity & 15600394 & 3 & 0.1 \\
\hline fumarase PA4333 & Citrate cycle & 15599529 & 41.3 & 1.7 \\
\hline phosphopantetheine adenylyltransferase CoaD & Cofactor and vitamin synthesis & 15595560 & 14.3 & 0.7 \\
\hline aconitate hydratase PA0794 & Citrate cycle & 15595991 & 32.7 & 1.7 \\
\hline $50 S$ ribosomal protein L3 RplC & Ribosome & 15599459 & 10.7 & 0.7 \\
\hline formyltetrahydrofolate deformylase PurU1 & Purine metabolism & 15599510 & 4.7 & 0.3 \\
\hline
\end{tabular}

PACs decreased the swarming motility of $P$. aeruginosa, both in the distance moved and the complexity of the swarming pattern.

We also found that PACs decreased biofilm produced by $P$. aeruginosa when applied continuously to the culture, which is consistent with published results in other Gram-negative bacteria such as $E$. coli and $P$. gingivalis [10-14]. Our experiments with cranberry PACs demonstrated a dose dependent reduction of preformed $P$. aeruginosa biofilm.
Several groups have found that P-fimbriae-mediated adherence to surfaces by $E$. coli is reduced by A-type PACs $[6,48]$. Based on our attachment studies, PACs do not significantly reduce the adherence of $P$. aeruginosa to surfaces. $P$. aeruginosa attachment may not be affected due to the fact that this organism contains no P-fimbriae related attachment mechanisms. Planktonic growth is reduced by high concentrations of PACs, and PACs-treatment appears to reduce the total number of $P$. aeruginosa within the biofilm. 


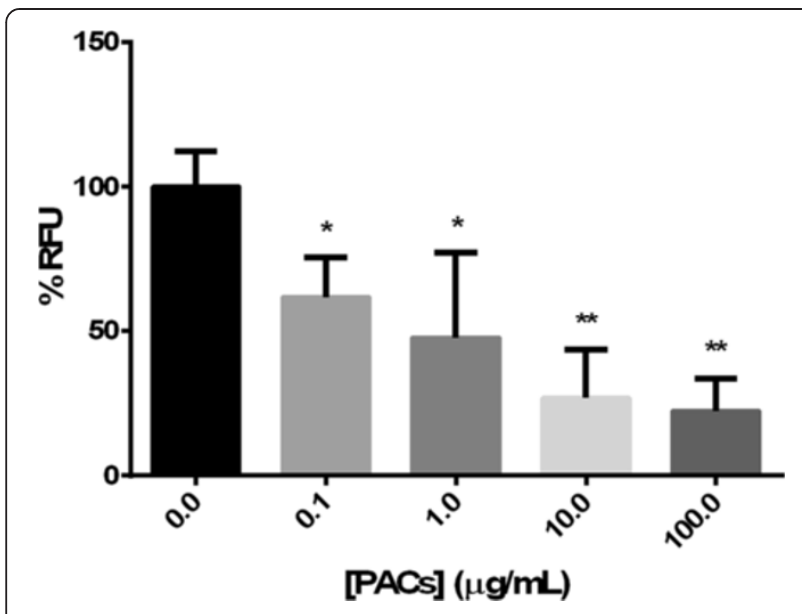

Figure 6 Resazurin reduction of $P$. aeruginosa in biofilm following cranberry PAC treatment. ${ }^{*}=p<0.05 ;{ }^{* *}=p<0.005$.

PACs have been shown to have an effect on a wide variety of bacterial systems, as their primary mechanism is iron chelation, an effect previously known in E. coli [9]. Proteomic analysis in this study shows that PAC treatment affects the abundance of many proteins in $P$. aeruginosa. Several of the proteins that are less abundant in the treated sample normally contain iron, such as cytochromes, suggesting a potential role of PAC iron chelation as a mechanism. Most notably, several of the up-regulated proteins are iron siderophores. In addition, $\mathrm{Fe}^{3+}$ is required for $P$. aeruginosa biofilms to fully mature into a large structure. It has been found that in iron-limiting conditions, $P$. aeruginosa will only form flat, thin biofilms [49], which we confirmed through fluorescent microscopy. Bacteria in these thin biofilms may be much less resistant to antibiotics and the host immune system than bacteria in a fully developed biofilm. Iron siderophores seem to be particularly effective as potentiators of aminoglycosides against $P$. aeruginosa, as it has been previously found that the activity of tobramycin was augmented when used in conjunction with lactoferrin [40].

Table 3 Checkerboard assay of gentamicin and PACs against $P$. aeruginosa, showing the decrease (potentiation) of the gentamicin MIC by the addition of PAC

\begin{tabular}{cccccccccc}
\hline \multicolumn{10}{c}{ [Gentamicin] $(\boldsymbol{\mu g} / \mathbf{m L})$} \\
\hline$[\mathrm{PAC}]$ & & 1.7 & 1.6 & 1.5 & 1.4 & 1.3 & 1.2 & 1.1 & 0 \\
$(\mu \mathrm{g} / \mathrm{mL})$ & 100 & 0.081 & 0.084 & 0.079 & 0.088 & 0.178 & 0.698 & 0.841 & 1.74 \\
& 10 & 0.066 & 0.066 & 0.069 & 0.067 & 0.093 & 0.439 & 0.693 & 1.746 \\
& 1 & 0.057 & 0.055 & 0.054 & 0.059 & 0.07 & 0.461 & 1.137 & 1.743 \\
& 0.1 & 0.108 & 0.056 & 0.053 & 0.06 & 0.056 & 0.903 & 1.687 & 1.774 \\
& 0.01 & 0.056 & 0.054 & 0.063 & 0.053 & $\mathbf{0 . 0 6 2}$ & 1.002 & 1.197 & 1.794 \\
& 0 & 0.054 & 0.061 & 0.055 & 0.241 & 0.647 & 1.267 & 1.727 & 1.806
\end{tabular}

Bold value indicates point of synergy.
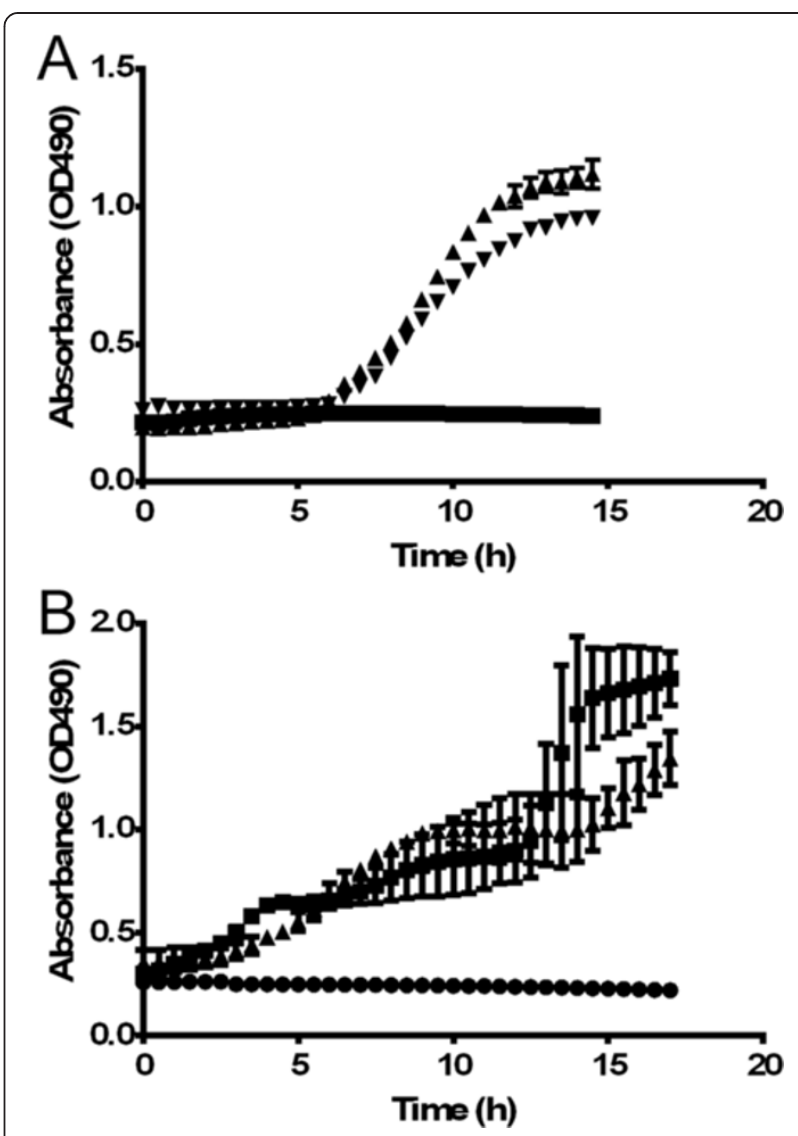

Figure 7 In vitro treatment of $P$. aeruginosa-infected mammalian cells with cranberry PACs. A. J774A.1 mouse macrophage infected cell line. B. HEk293T/17 human kidney epithelial cell line. $\boldsymbol{\Delta}$ = untreated; $\boldsymbol{\nabla}=$ treated with PACs; $\mathbf{\square}=$ no bacteria control.

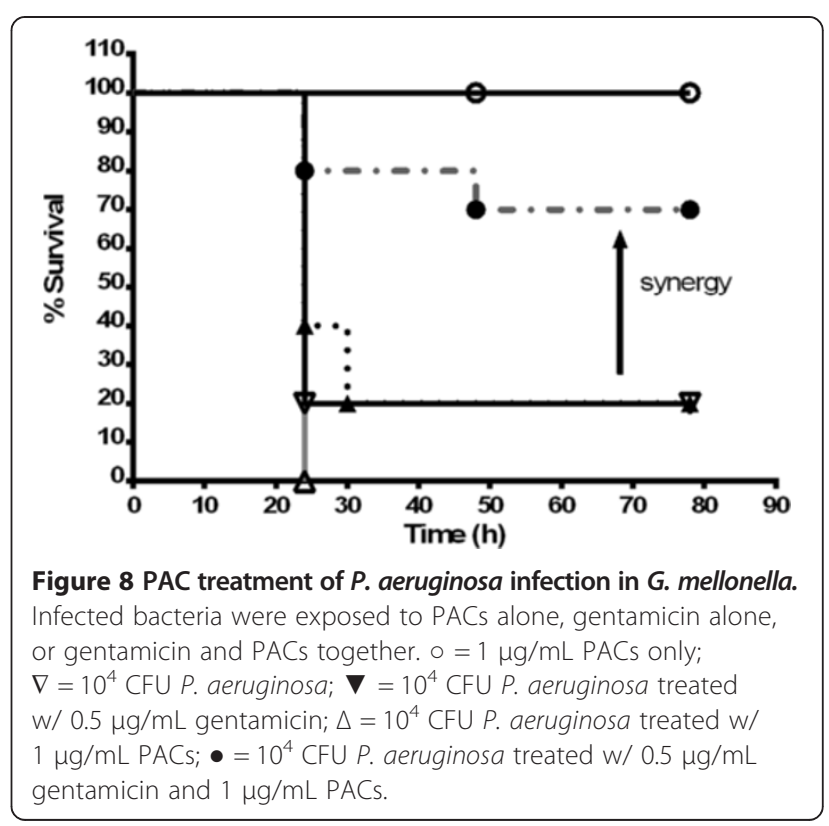


We performed a series of experiments to explore how PACs may affect the action of a traditional antibiotic such as gentamicin. In vitro experiments showed the potentiation effect of PACs on the MIC of gentamicin. This was supported by in vivo studies in the G. mellonella model which demonstrated that a low dose of gentamicin with PACs prolonged survival in $P$. aeruginosa-infected worms significantly more than antibiotic or PACs alone. Thus cranberry PACs may be acting as antibiotic adjuvant for the action of gentamicin [50]. Cranberry juice has long been used as a prophylactic for urinary tract bacterial infection, and the use of PACs should be further explored to potentiate the action of antibiotics and reduce bacterial colonization and biofilm development during other infections caused by $P$. aeruginosa.

\section{Additional file}

Additional file 1: Table S1. All up- and down-regulated proteins in

PAC treated $P$. aeruginosa bacteria as determined by LC/MS/MS.

\section{Competing interests}

The authors declare that they have no competing interests.

\section{Authors' contributions}

RKU and SMB performed experiments, analyzed data, and contributed the manuscript. WZ performed mass spectrometry, analyzed data, and contributed to the manuscript. MVH conceived the study and contributed to the manuscript. All authors approved the final manuscript.

\section{Acknowledgments}

We acknowledge A. Ahmed and R. Brandt for their role in early experiments on this project and S. Dean for helpful discussions. We are grateful to A. Nwabueze for assisting with the confocal microscopy and to Dr. A. Howell, Rutgers University for providing PACS. Many thanks to J. Roman for assistance with cell culture. RKU received an Undergraduate Research Apprenticeship from George Mason University. SMB and MVH are supported by HDTRA1-12-C-0039 Translational Peptide Research for Personnel Protection. Publication of this article is supported by the George Mason University Open Access Fund.

\section{Author details}

'Department of Biology, George Mason University, Manassas, Virginia, USA. ${ }^{2}$ School of Systems Biology, George Mason University, Manassas, Virginia, USA. ${ }^{3}$ Center for Applied Proteomics and Molecular Medicine, George Mason University, Manassas, Virginia, USA. ${ }^{4}$ National Center for Biodefense and Infectious Diseases, George Mason University, Manassas, Virginia, USA.

Received: 27 April 2014 Accepted: 11 December 2014

Published: 16 December 2014

\section{References}

1. Flemming HC, Wingender J: The biofilm matrix. Nat Rev Microbiol 2010, 8(9):623-633.

2. Costerton JW, Stewart PS, Greenberg EP: Bacterial biofilms: a common cause of persistent infections. Science 1999, 284(5418):1318-1322.

3. Kuzminski LN: Cranberry juice and urinary tract infections: is there a beneficial relationship? Nutr Rev 1996, 54(11 Pt 2):S87-S90.

4. Fleet JC: New support for a folk remedy: cranberry juice reduces bacteriuria and pyuria in elderly women. Nutr Rev 1994, 52(5):168-170.

5. Borukh IF, Kirbaba VI, Senchuk GV: [Antimicrobial properties of cranberry]. Vopr Pitan 1972, 31(5):82.

6. Foo LY, Lu Y, Howell AB, Vorsa N: A-Type proanthocyanidin trimers from cranberry that inhibit adherence of uropathogenic P-fimbriated Escherichia coli. J Nat Prod 2000, 63(9):1225-1228.
7. Howell AB, Reed JD, Krueger CG, Winterbottom R, Cunningham DG, Leahy M: A-type cranberry proanthocyanidins and uropathogenic bacterial anti-adhesion activity. Phytochemistry 2005, 66(18):2281-2291.

8. Bodet C, Piche M, Chandad F, Grenier D: Inhibition of periodontopathogenderived proteolytic enzymes by a high-molecular-weight fraction isolated from cranberry. J Antimicrob Chemother 2006, 57(4):685-690.

9. Hidalgo G, Ponton A, Fatisson J, O'May C, Asadishad B, Schinner T, Tufenkji $\mathrm{N}$ : Induction of a state of iron limitation in uropathogenic Escherichia coli CFT073 by cranberry-derived proanthocyanidins as revealed by microarray analysis. Appl Environ Microbiol 2011, 77(4):1532-1535.

10. Ahuja S, Kaack B, Roberts J: Loss of fimbrial adhesion with the addition of Vaccinum macrocarpon to the growth medium of P-fimbriated Escherichia coli. J Urol 1998, 159(2):559-562.

11. Sobota AE: Inhibition of bacterial adherence by cranberry juice: potential use for the treatment of urinary tract infections. J Urol 1984, 131(5):1013-1016.

12. Tapiainen T, Jauhiainen H, Jaakola L, Salo J, Sevander J, Ikaheimo I, Pirttila AM, Hohtola A, Uhari M: Biofilm formation and virulence of uropathogenic Escherichia coli in urine after consumption of cranberry-lingonberry juice. Eur J Clin Microbiol Infect Dis 2012, 31(5):655-662.

13. Yamanaka A, Kimizuka R, Kato T, Okuda K: Inhibitory effects of cranberry juice on attachment of oral streptococci and biofilm formation. Oral Microbiol Immunol 2004, 19(3):150-154.

14. Yamanaka A, Kouchi T, Kasai K, Kato T, Ishihara K, Okuda K: Inhibitory effect of cranberry polyphenol on biofilm formation and cysteine proteases of Porphyromonas gingivalis. J Periodontal Res 2007, 42(6):589-592.

15. Amer LS, Bishop BM, van Hoek ML: Antimicrobial and antibiofilm activity of cathelicidins and short, synthetic peptides against Francisella. Biochem Biophys Res Commun 2010, 396(2):246-251.

16. O'May C, Ciobanu A, Lam H, Tufenkji N: Tannin derived materials can block swarming motility and enhance biofilm formation in Pseudomonas aeruginosa. Biofouling 2012, 28(10):1063-1076.

17. Bonifait L, Grenier D: Cranberry polyphenols: potential benefits for dental caries and periodontal disease. J Can Dent Assoc 2010, 76:a130.

18. Duarte S, Gregoire S, Singh AP, Vorsa N, Schaich K, Bowen WH, Koo H: Inhibitory effects of cranberry polyphenols on formation and acidogenicity of Streptococcus mutans biofilms. FEMS Microbiol Lett 2006, 257(1):50-56.

19. Matsushima M, Suzuki T, Masui A, Kasai K, Kouchi T, Takagi A, Shirai T, Mine T: Growth inhibitory action of cranberry on Helicobacter pylori. J Gastroenterol Hepatol 2008, 23(Suppl 2):S175-S180.

20. Yamanaka-Okada A, Sato E, Kouchi T, Kimizuka R, Kato T, Okuda K: Inhibitory effect of cranberry polyphenol on cariogenic bacteria. Bull Tokyo Dent Coll 2008, 49(3):107-112.

21. La VD, Labrecque J, Grenier D: Cytoprotective effect of proanthocyanidin-rich cranberry fraction against bacterial cell wall-mediated toxicity in macrophages and epithelial cells. Phytother Res 2009, 23(10):1449-1452.

22. Toivanen M, Ryynanen A, Huttunen S, Duricova J, Riihinen K, Torronen R, Lapinjoki S, Tikkanen-Kaukanen C: Binding of Neisseria meningitidis pili to berry polyphenolic fractions. J Agric Food Chem 2009, 57(8):3120-3127.

23. Pseudomonas aeruginosa: Infections and Treatment, Volume 12. New York: Informa Health Care; 1994.

24. Ciofu O, Hansen CR, Hoiby N: Respiratory bacterial infections in cystic fibrosis. Curr Opin Pulm Med 2013, 19(3):251-258.

25. Dean SN, Bishop BM, van Hoek ML: Susceptibility of pseudomonas aeruginosa biofilm to alpha-helical peptides: D-enantiomer of LL-37. Front Microbiol 2011, 2:128.

26. Tremblay J, Deziel E: Improving the reproducibility of Pseudomonas aeruginosa swarming motility assays. J Basic Microbiol 2008, 48(6):509-515.

27. Durham-Colleran MW, Verhoeven AB, van Hoek ML: Francisella novicida forms in vitro biofilms mediated by an orphan response regulator. Microb Ecol 2010, 59(3):457-465.

28. Dean SN, Bishop BM, van Hoek ML: Natural and synthetic cathelicidin peptides with anti-microbial and anti-biofilm activity against Staphylococcus aureus. BMC Microbiol 2011, 11:114.

29. OToole GA: Microtiter dish biofilm formation assay. J Visual Exp 2011, 47:2437.

30. Ahmad S, Hunter L, Qin A, Mann BJ, van Hoek ML: Azithromycin effectiveness against intracellular infections of Francisella. BMC Microbio/ 2010, 10:123.

31. Hall MJ, Middleton RF, Westmacott D: The fractional inhibitory concentration (FIC) index as a measure of synergy. J Antimicrob Chemother 1983, 11(5):427-433. 
32. Lomovskaya O, Warren MS, Lee A, Galazzo J, Fronko R, Lee M, Blais J, Cho D, Chamberland S, Renau T, Leger R, Hecker S, Watkins W, Hoshino K, Ishida H, Lee VJ: Identification and characterization of inhibitors of multidrug resistance efflux pumps in Pseudomonas aeruginosa: novel agents for combination therapy. Antimicrob Agents Chemother 2001, 45(1):105-116.

33. Tolker-Nielsen T, Sternberg C: Growing and Analyzing Biofilms in Flow Chambers. In Current Protocols in Microbiology. Hoboken, NJ: John Wiley \& Sons, Inc; 2005

34. Zhou W, Capello M, Fredolini C, Racanicchi L, Piemonti L, Liotta LA, Novelli F, Petricoin EF: Proteomic analysis reveals Warburg effect and anomalous metabolism of glutamine in pancreatic cancer cells. J Proteome Res 2012, 11(2):554-563.

35. Elias JE, Gygi SP: Target-decoy search strategy for increased confidence in large-scale protein identifications by mass spectrometry. Nat Methods 2007, 4(3):207-214

36. McKenney ES, Sargent M, Khan H, Uh E, Jackson ER, San Jose G, Couch RD, Dowd CS, van Hoek ML: Lipophilic prodrugs of FR900098 are antimicrobial against Francisella novicida in vivo and in vitro and show GlpT independent efficacy. PLoS One 2012, 7(10):e38167.

37. O'Loughlin CT, Miller LC, Siryaporn A, Drescher K, Semmelhack MF, Bassler BL: A quorum-sensing inhibitor blocks Pseudomonas aeruginosa virulence and biofilm formation. Proc Natl Acad Sci U S A 2013, 110(44):17981-17986.

38. Wu H, Song Z, Hentzer M, Andersen JB, Molin S, Givskov M, Hoiby N: Synthetic furanones inhibit quorum-sensing and enhance bacterial clearance in Pseudomonas aeruginosa lung infection in mice. J Antimicrob Chemother 2004, 53(6):1054-1061.

39. Lin B, Johnson BJ, Rubin RA, Malanoski AP, Ligler FS: Iron chelation by cranberry juice and its impact on Escherichia coli growth. Biofactors (Oxford, England) 2011, 37(2):121-130.

40. Andres MT, Viejo-Diaz M, Perez F, Fierro JF: Antibiotic tolerance induced by lactoferrin in clinical Pseudomonas aeruginosa isolates from cystic fibrosis patients. Antimicrob Agents Chemother 2005, 49(4):1613-1616.

41. Woolfrey BF, Lally RT, Ederer MN, Quall CO: Evaluation of the automicrobic system for susceptibility testing of Pseudomonas aeruginosa to gentamicin, tobramycin, and amikacin. J Clin Microbiol 1984, 19(4):502-505.

42. Koo H, Duarte S, Murata RM, Scott-Anne K, Gregoire S, Watson GE, Singh AP, Vorsa N: Influence of cranberry proanthocyanidins on formation of biofilms by Streptococcus mutans on saliva-coated apatitic surface and on dental caries development in vivo. Caries Res 2010, 44(2):116-126.

43. Leshem R, Maharshak I, Ben Jacob E, Ofek I, Kremer I: The effect of nondialyzable material (NDM) cranberry extract on formation of contact lens biofilm by Staphylococcus epidermidis. Invest Ophthalmol Vis Sci 2011, 52(7):4929-4934.

44. Feldman M, Weiss El, Ofek I, Steinberg D: Interference of cranberry constituents in cell-cell signaling system of Vibrio harveyi. Curr Microbiol 2009, 59(4):469-474

45. Labrecque J, Bodet C, Chandad F, Grenier D: Effects of a high-molecularweight cranberry fraction on growth, biofilm formation and adherence of Porphyromonas gingivalis. J Antimicrob Chemother 2006, 58(2):439-443.

46. Polak D, Naddaf R, Shapira L, Weiss El, Houri-Haddad Y: Protective potential of non-dialyzable material fraction of cranberry juice on the virulence of P. gingivalis and F. nucleatum mixed infection. J Periodontol 2013, 84(7):1019-1025

47. O'May C, Tufenkji N: The swarming motility of Pseudomonas aeruginosa is blocked by cranberry proanthocyanidins and other tannin-containing materials. Appl Environ Microbiol 2011, 77(9):3061-3067.

48. Foo LY, Lu Y, Howell AB, Vorsa N: The structure of cranberry proanthocyanidins which inhibit adherence of uropathogenic P-fimbriated Escherichia coli in vitro. Phytochemistry 2000, 54(2):173-181.

49. Cai Y, Wang R, An MM, Liang BB: Iron-Depletion prevents biofilm formation in Pseudomonas Aeruginosa through twitching mobility and quorum sensing. Brazilian J Microbiol 2010, 41(1):37-41.

50. Farha MA, Brown ED: Discovery of antibiotic adjuvants. Nat Biotechnol 2013, 31(2):120-122

doi:10.1186/1472-6882-14-499

Cite this article as: Ulrey et al:: Cranberry proanthocyanidins have anti-biofilm properties against Pseudomonas aeruginosa. BMC Complementary and Alternative Medicine 2014 14:499.

\section{Submit your next manuscript to BioMed Central and take full advantage of:}

- Convenient online submission

- Thorough peer review

- No space constraints or color figure charges

- Immediate publication on acceptance

- Inclusion in PubMed, CAS, Scopus and Google Scholar

- Research which is freely available for redistribution 